\title{
Displaying Genealogy with Adoptions and Multiple Remarriages Using the WHIteBasE
}

\author{
Seiji Sugiyama ${ }^{1}$, Atsushi Ikuta ${ }^{2}$, Daisuke Yokozawa ${ }^{2}$, \\ Miyuki Shibata ${ }^{2}$, and Tohru Matsuura ${ }^{3}$ \\ 1 Info. Science \& Engineering, Ritsumeikan University, Kusatsu, Shiga, Japan \\ seijisan@is.ritsumei.ac.jp \\ 2 Human Informatics, Otani University, Kyoto, Japan \\ a.ikuta@sch.otani.ac.jp, dyokozawa@gmail.com, neko@res.otani.ac.jp \\ 3 Hokkaido University Hospital, Sapporo, Hokkaido, Japan \\ macchan@med.hokudai.ac.jp
}

\begin{abstract}
In this research, needs of displaying genealogy with various family relations are described and those solutions using our WHIteBasE method are proposed. Previous WHIteBasE method has perfectly been able to integrate each relation that includes a married couple and their children, and has been able to display complex relations with segment intersections easily. It has also been added that Genealogy with Direct Segments (DS), Genealogy with Hooked Segments (HS) and Annotation Data Always Displayed (ADAD). In this paper, two new functions are added to the WHIteBasE method. One is 'Genealogy with Adoptions'. Using 'Adopted Segments (AS)', not only biological family relations but also social family relations can be displayed simultaneously. The other is 'Genealogy with Multiple Remarriages'. Using 'Double Bends (DB)', crossing relations more than $3 \times 3$ both sexes can be displayed perfectly. Our improved software that can display AS and DB automatically and seamlessly by only mouse operation is presented.
\end{abstract}

Keywords: Adoptions, Multiple Remarriages, Hidden Node, Segment Intersections, Search Algorithm, Free Layout.

\section{Introduction}

There is a requirement to display complex relations in family trees. However, it cannot be displayed perfectly in existing genealogy display software [1]-20]. When complex relations are inputted in the software, one individual is often displayed in multiple places automatically. It is thought that almost all of them consider only simple family trees and have no idea to display complex layout. As a result, users must reconstruct the scattered individual placements into only one relation in their mind, though it is graphical display software. On the other hand, 3D graphics is rather more difficult to understand complex relations than $2 \mathrm{D}$ because many names and segments overlap 21. GEDCOM 22, a de facto standard for recording genealogy data exchange format, is not enough to display complex relations because it considers no layout information. 


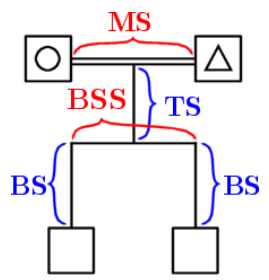

Fig. 1. Segment Names for Japanese Regular Layout

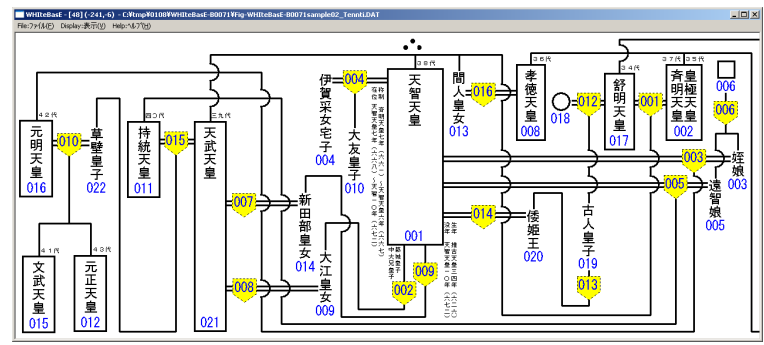

Fig. 2. Our Previous Software 24]

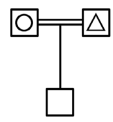

(a) a child

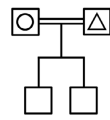

(b) children

Fig. 3. Regular Layout

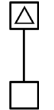

(a) a child (b) children

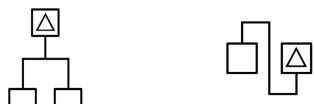

(a) a child

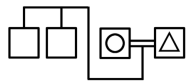

(b) children

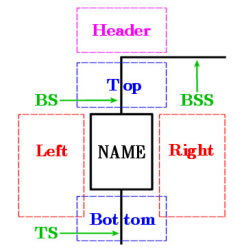

Fig. 9. Five Areas

(b) children

Fig. 4. DS from a Single Parent

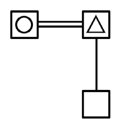

(a) a child

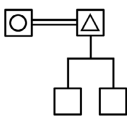

(b) children

Fig. 5. DS from Parents

Fig. 7. HS from a Single Parent

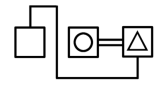

(a) a child

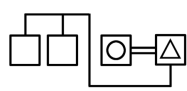

(b) children
Fig. 8. HS from Parents

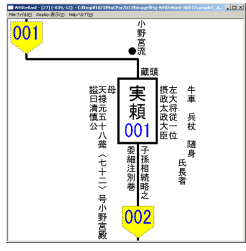

Fig. 10. $A D A D$

New software has been constructed in our research so that it can display complex relations 23. Our software uses an event oriented data management method, "WHIteBasE" (Widespread Hands to InTErconnect BASic Elements). It is a hidden node for integrating relations that include a married couple and their children. If WHIteBasE is used, one individual can be displayed only once because complex relations with segment intersections can be displayed. Intuitive inputs and inspections such as map image display systems can be realized.

In our previous software, the regular Japanese layout style has been used as shown in Fig. 1. It includes a double horizontal segment MS (Marriage Segment), a vertical segment TS (Trunk Segment), a horizontal segment BSS (Brothers and Sisters Segment), and a vertical segment BS (Branch Segment). The ' $\triangle$ ' denotes a male and the ' $O$ ' denotes a female that are a couple connected by using MS. Children are connected from the intermediate of MS by using TS, BSS and BS.

Fig. 2] shows our previous software 24. It has been constructed by using not only 'Regular Layout' as shown in Fig. 3 but also 'Genealogy with Direct Segments (DS)' as shown in Figs.45. 'Genealogy with Hooked Segments (HS)' as shown in Figs.668, and 'Annotation Data Always Displayed (ADAD)' as shown 


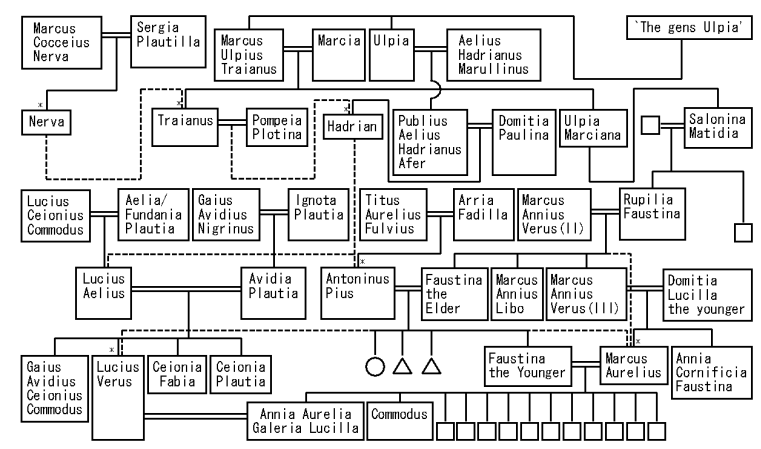

Solid Segments: Biological Relations Dashed Segments: Social Family Relations

Fig. 11. Sample of Genealogy with Adoptions 25]<smiles>[GeH3]O[GeH3]</smiles>

(a) a child

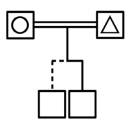

(a)

Fig. 15. AS from MS in HS

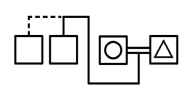

(d)

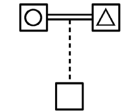

(a) a child

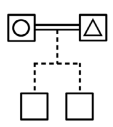

(b) children

Fig. 12. AS from MS

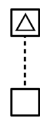

(a) a child

(b) children

Fig. 13. AS from a Single Parent<smiles>O[Hg]</smiles>

(a) a child

(b) children

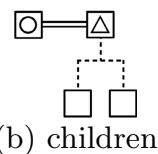

Fig. 14. AS from Parents

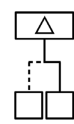

(b)

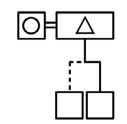

(c)

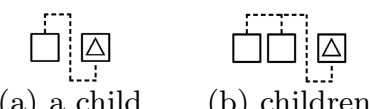

(a) a child

(b) children

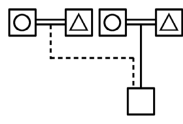

(g)

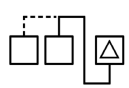

(e)

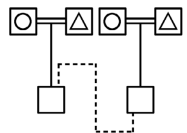

(h)

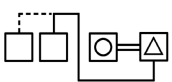

(f)

Fig. 16. AS from a Single Parent in HS

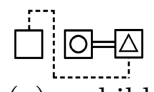

(a) a child

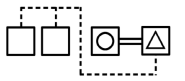

(b) children

Fig. 17. AS from Parents in HS

Fig. 18. Biological Relations and Adoptions

in Fig.9 10, Regular layout, DS, HS and ADAD can be displayed automatically and seamlessly by only mouse operations.

In this paper, needs of two more different family relations are described and those solutions are proposed. One is 'Genealogy with Adoptions' such as shown in Fig.11]25]. Not only 'Biological Relations' showing solid segments but also 'Social Family Relations' such as adoptions showing dashed segments are often written simultaneously. It is necessary to display these dashed segments named 'Adopted Segments (AS)' for connecting adoptions. Connections of AS from parent(s) to a child/children have three types as the following:

(A) AS from MS: One married couple is connected with a child/children from the intermediate of MS as shown in Figs.12(a),(b).

(B) AS from a Single Parent: Only a single parent is connected with a child/children directly without using MS as shown in Figs.13(a),(b).

(C) AS from Parents: Only a single parent that has the other parent using MS is connected with a child/children directly as shown in Figs.14(a),(b). 


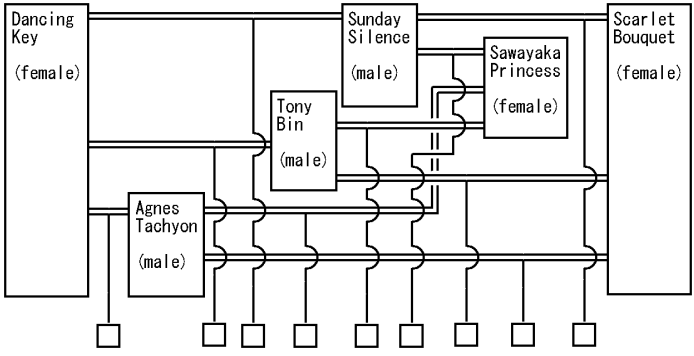

Fig. 19. Sample of Multiple Remarriages [26]

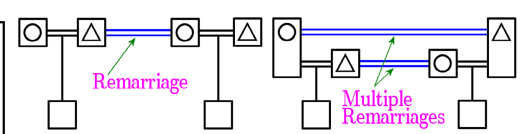

(a) Regular

(b) Multiple

Fig. 20. Type of Remarriages

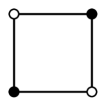

(a) $2 \times 2$

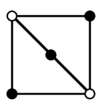

(b) $2 \times 3$

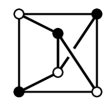

(c) $3 \times 3$
Fig. 21. Connection Models

On the other hand, connections of AS from parent(s) to a child/children in HS have three types as the following:

(a) AS from MS in HS: One married couple is connected with a child/children from the intermediate of MS as shown in Figs.15(a),(b).

(b) AS from a Single Parent in HS: Only a single parent is connected with a child/children directly without using MS as shown in Figs.16(a),(b).

(c) AS from Parents in HS: Only a single parent that has the other parent using MS is connected with a child/children directly as shown in Figs.17(a),(b).

The (A)-(C) and (a)-(c) are similar to DS and HS respectively (Fig. 3 8 ). If all descendants in a family are adoptions, the difference is only a change of segment types from solid segments to dashed segments. On the other hand, if a family has children including both biological relations and adoptions, the segment type of TS becomes the solid segment, and both solid segments and dashed segments are used on the way to the children as shown in Figs.18(a)-(f). If there are two families, one is biological relations and the other is adoptions, and/or if there is a connection from one of brothers and sisters to others, both solid segments and dashed segments in HS are used as shown in Figs.18(g)-(i).

The other is 'Genealogy with Multiple Remarriages' such as mating of the racehorses shown in Fig.19. In this case, a bloodline is important. As a result, there is a case that relations including only several horses grow very powerful, because of focusing on a strong horse. Connections of remarriages have two types as the following:

(I) Regular Remarriages: A married person marries again after her husband or his wife has died or after being divorced as shown in Fig.20(a). In this case, there is no closed areas by MS and individuals' text boxes.

(II) Multiple Remarriages: All persons of plural couples marry again by replacing each other as shown in Fig.20(b). In this case, there are closed areas.

Closed areas cause crossing of MS (Fig. 19). In our previous research, no crossing of MS has been considered, and the horizontal MS style has been only defined. To cope with the difficulty, 'Double Bends (DB)' for crossing of MS is added.

Fig.21(a) shows the connection model of the minimum unit of closed areas by $2 \times 2$ partners (two males and two females). The ' $\bullet$ ' and the ' $O$ ' denote a sexual difference. Figs. 22(a)-(d) show genealogy style of the connections 2x2. In this case, 


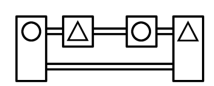

(a)

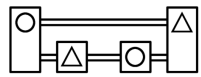

(b)

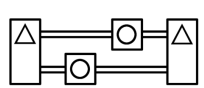

(c)

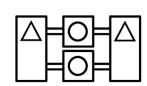

(d)

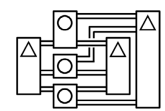

(a)

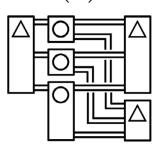

(c) (d)

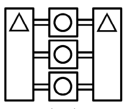

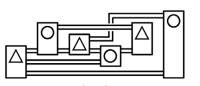

(b)

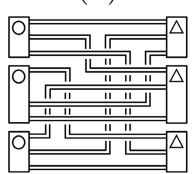

(d)

(a)

(b)

(c)

Fig. 23. Connections $2 \times 3$

no crossing of MS is occurred. Fig.21(b) shows the connection model of closed areas by 2x3 partners. Figs.23(a)-(d) show genealogy style of the connections $2 \times 3$. In this case, no crossing of MS is also occurred. On the other hand, Fig. 21)(c) shows the connection model of closed areas by 3x3 partners. Figs.24(a)-(d) show genealogy style of the connections $3 \times 3$. In this case, a crossing of MS is occurred and it is necessary to display DB.

These kinds of family relations including AS and DB cannot be displayed in the existing software. Because almost all of them focus on no adoptions and no multiple remarriages. Therefore, it is necessary to construct new software.

In this research, an upgrade, adding two kinds of family relations that use AS and DB to our previous genealogy display software by using the WHIteBasE method, is proposed. Our improved software that can also display AS and DB automatically and seamlessly by only mouse operations is presented.

\section{WHIteBasE}

In this section, the WHIteBasE method that is our previous proposal [23] 24 . is briefly introduced. A relation between a married couple and their child is managed as an event by a Hidden Node, WHIteBasE as shown in Fig.25(a). The connection model using WHIteBasE is shown in Fig.25(b). WHIteBasE has three keyholes, $S_{L}, S_{R}$ (Substance) and $D$ (Descendant). Individuals have two keys, $A$ (Ascendant) and $M$ (Marriage). $A$ can connect with $D$, and $M$ can connect with $S_{L}$ or $S_{R}$, where denote one family.

For plural children as shown in Fig.26(a), $D$ is extended in multiple keyholes $D_{j}$ as shown in Fig.26(b). For multiple marriages as shown in Fig.27(a), $M$ is extended in multiple keys $M_{k}$ (Fig.27(b)) and plural WHIteBasEs are used. That can define all of biological relations perfectly.

A set of $W_{i}$ that defines WHIteBasEs and a set of $I_{j}$ that defines Individual Nodes are represented by

$$
\begin{gathered}
W_{i}=\left\{S_{L}, S_{R}, D_{j}, \mathbf{Q}\right\} \\
I_{j}=\left\{A, M_{k}\right\}
\end{gathered} \quad\left\{\begin{array}{l}
i=0,1, \cdots, i_{\max } \\
j=0,1, \cdots, j_{\max } \\
k=0,1, \cdots, k_{\max }
\end{array}\right.
$$

where $i, j, k$ denote IDs on the data table respectively, $i_{\max }, j_{\max }, k_{\max }$ are the maximum values, $S_{L}, S_{R}$ denote IDs of a couple, $D_{j}$ denotes ID of descendants, 


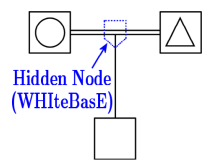

(a) Genealogy Style

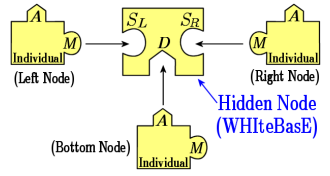

(b) Model of WHIteBasE

Fig. 25. Connection for a married couple and a child

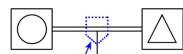

WHIteBasE

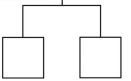

(a) Genealogy Style

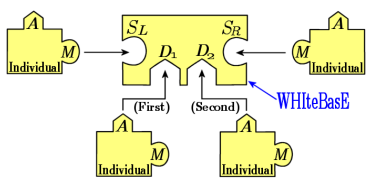

(b) Model of WHIteBasE

Fig. 26. Connection for a married couple and children

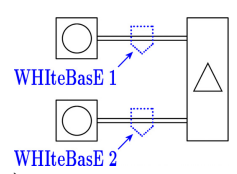

(a) Genealogy Style

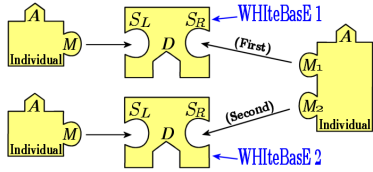

(b) Model of WHIteBasE

Fig. 27. Connection for multiple marriages

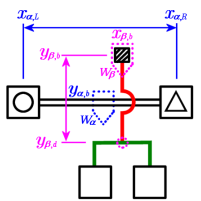

(a) Pattern 1

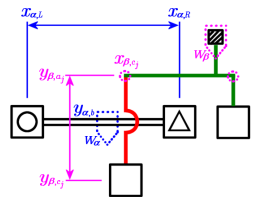

(b) Pattern 2

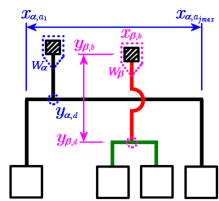

(c) Pattern 3

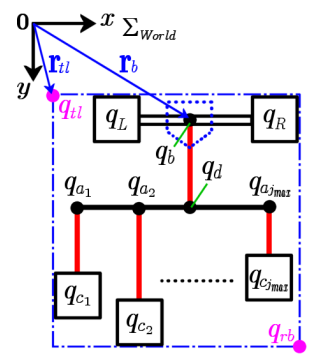

Fig. 28. Coordinate System

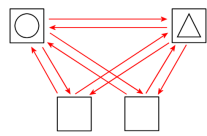

(a) Existing Method

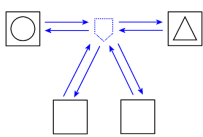

(b) WHIteBasE Method

Fig. 29. Number of references

Fig. 30. Search Method for Segment Intersections

$A$ denotes ID of an ascendant WHIteBasE, and $M_{k}$ denotes ID of WHIteBasEs for handling marriages. Individuals are managed by using data table including names and annotation data. WHIteBasEs are managed by using data table separated from Individuals. Remarriages are managed by using plural WHIteBasEs. $\mathbf{Q}$ denotes a set of coordinate values of each position managed by a WHIteBasE measured from the origin of the displaying area (Fig.28) and is represented by

$$
\mathbf{Q}=\left\{q_{b}, q_{L}, q_{R}, q_{d}, q_{a_{j}}, q_{c_{j}}, q_{t l}, q_{r b}\right\} .
$$

where $q_{b}$ denotes a WHIteBasE's position, $q_{L}, q_{R}$ denote the parents' positions, $q_{d}$ denotes a junction's position between MS and TS, $q_{c_{j}}$ denotes children's positions, $q_{a_{j}}$ denotes junctions' positions between BSS and BS, $q_{t l}, q_{r b}$ denote positions of top-left and bottom-right of all area managed by a WHIteBasE.

One of advantages using WHIteBasE is the decreased reference volume. Using the existing software, all of individuals connect with other individuals (Fig.29(a)). On the other hand, using the WHIteBasE method, two reference links per a child decrease (Fig.29(b)). Moreover, the user can understand the complex relations intuitively and can input and inspect them easily. 


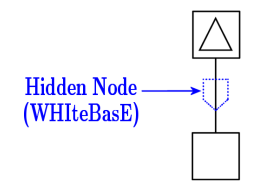

(a) Single Parent

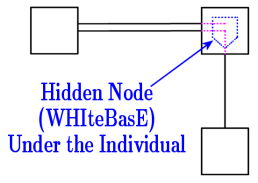

(b) Parents

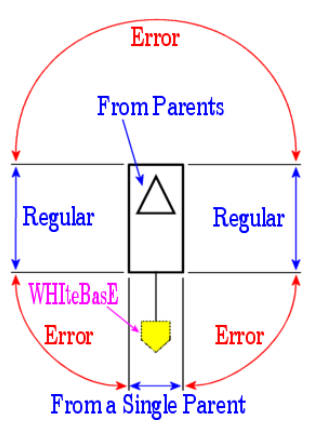

(c) Switching Mode

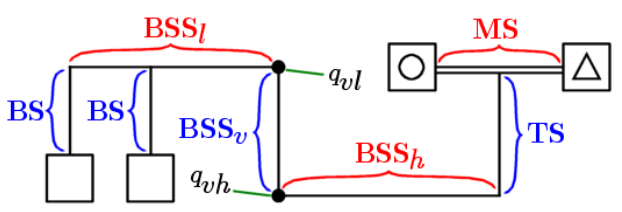

(a) Names

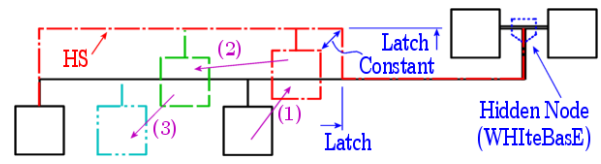

(b) Switching Mode

Fig. 31. Genealogy with Direct Segments Fig. 32. Genealogy with Hooked Segments

Using segment intersections is necessary for displaying complex relations. If only one WHIteBasE is used, there is no segment intersections. On the other hand, if plural WHIteBasEs are used and horizontal segments (MS, BSS) and vertical segments (TS, BS) are displayed, the positions of segment intersections can be calculated by using only four patterns of line crossing as the following:

(a) MS of $W_{\alpha}$ and TS of $W_{\beta}$ are crossing (Fig. 30(a))

If $x_{\alpha, L}<x_{\beta, b}<x_{\alpha, R} \& y_{\beta, b}<y_{\alpha, b}<y_{\beta, d}$, the intersection is $\left(x_{\beta, b}, y_{\alpha, b}\right)$.

(b) MS of $W_{\alpha}$ and BS of $W_{\beta}$ are crossing (Fig. 30(b))

If $x_{\alpha, L}<x_{\beta, c_{j}}<x_{\alpha, R} \& y_{\beta, a_{j}}<y_{\alpha, b}<y_{\beta, c_{j}}$, the intersection is $\left(x_{\beta, c_{j}}, y_{\alpha, b}\right)$.

(c) BSS of $W_{\alpha}$ and TS of $W_{\beta}$ are crossing (Fig. 30(c))

If $x_{\alpha, a_{1}}<x_{\beta, b}<x_{\alpha, a_{\max }} \& y_{\beta, b}<y_{\alpha, d}<y_{\beta, d}$, the intersection is $\left(x_{\beta, b}, y_{\alpha, d}\right)$.

(d) BSS of $W_{\alpha}$ and BS of $W_{\beta}$ are crossing (Fig. 30(d))

If $x_{\alpha, a_{1}}<x_{\beta, c_{j}}<x_{\alpha, a_{j_{\text {max }}}} \& y_{\beta, a_{j}}<y_{\alpha, d}<y_{\beta, c_{j}}$, the intersection is $\left(x_{\beta, c_{j}}, y_{\alpha, d}\right)$.

The four patterns are only calculated while only two WHIteBasEs' management rectangle $q_{t l}, q_{r b}$ are overlapped. Therefore, this search method is faster than checking all segments.

Genealogy with Direct Segments (DS) is necessary for connecting parent(s) and their child directly. DS from a Single Parent is used while WHIteBasE lies under a parent (Fig.31(a)). DS from Parents is used while WHIteBasE overlapped one of parents (Fig.31(b)). Two kinds of DS are automatically switched according to the positions only (Fig.31(c)) without changing the connection model [24]. Genealogy with Hooked segment (HS) is necessary for connecting parents and their children in parallel layouts. BSS as shown in Fig.11 is extended to three parts; $\mathrm{BSS}_{l}, \mathrm{BSS}_{v}$, and $\mathrm{BSS}_{h}$ (Fig.32(a)). For calculating HS, positions $q_{v l}, q_{v h}$ are added to Eq. (2). Occurring HS is automatically switched according to the positions only (Fig. 32 (b)) 24].

The search method of segment intersections for DS and HS has been proposed in our previous paper 24. Therefore, the figures of search patterns are omitted in this paper. Only nine search patterns are used for DS and HS because there are three horizontal segments and three vertical segments. 


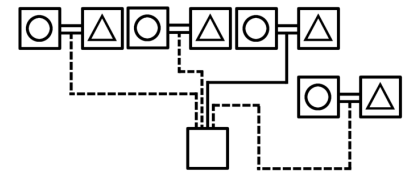

(a) Genealogy Style

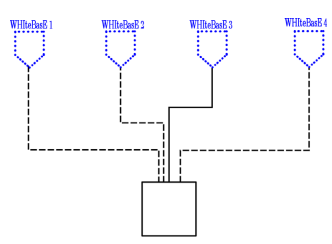

(b) Hidden Node Connection

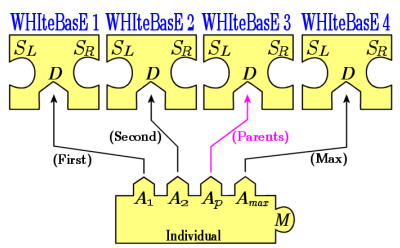

(c) Model of WHIteBasE

Fig. 33. Connection Model for Genealogy with Adoptions

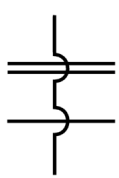

(a)

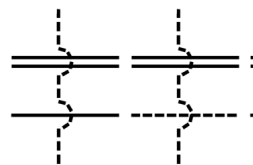

(b)

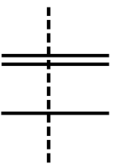

(d)

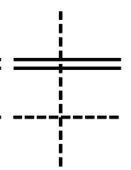

(e)

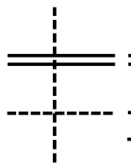

(f)

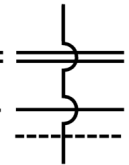

(g)

Fig. 34. Segment Intersections using AS

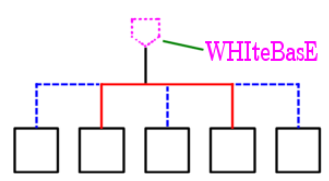

(a) Regular Layout

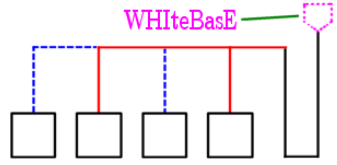

(b) Using HS

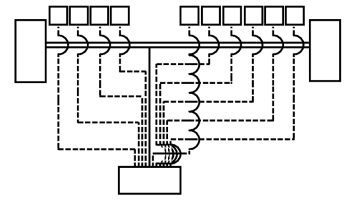

Fig. 35. AS and Arc

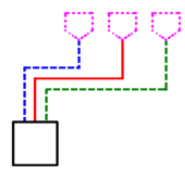

(c) Multiple

Fig. 36. Displaying Method for AS

\section{Genealogy with Adoptions}

Adoptions have not only biological parents but also other plural parents as shown in Figs.33(a),(b). Therefore, the ascendant key $A$ in the Individual is extended in multiple keys $A_{l}\left(l=0,1, \cdots, p, \cdots, l_{\max }\right)$ as shown in Fig.33(c), where $l$ denotes the IDs on the data table, $p$ denotes the ID of biological parents' WHIteBasE, $l_{\max }$ denotes the maximum value, respectively. If $l=p$, use the solid segments. If $l \neq p$, use the dashed segments AS.

Consider the arc style of segment intersections in the case of using AS. It is necessary to use arcs on the intersection between solid horizontal segments and solid vertical segments as shown in Fig.34(a). It can be displayed to use arcs on the intersection between solid horizontal segments and dashed vertical segments as shown in Fig.34(b). However, the dashed segments are broken up if arcs are used in the intersection between dashed horizontal segments and dashed vertical segments as shown in Fig.34(c). In addition, it is difficult to display arcs in the narrow area, especially near the area connecting with AS as shown in Fig.35. Therefore, it is useful that no arcs is used for crossing by using AS as shown in Figs. $34(\mathrm{~d})-(\mathrm{g})$.

The search method of segment intersections for AS is very simple because AS has no arcs. Classify only solid segment sections and dashed segment sections as shown in Figs.36(a),(b). Then, search the segment intersections for only solid sections. The connecting points of AS on the top of Individual text box is line up in order to locate WHIteBasE's horizontal positions as shown in Fig. 36)(c). 


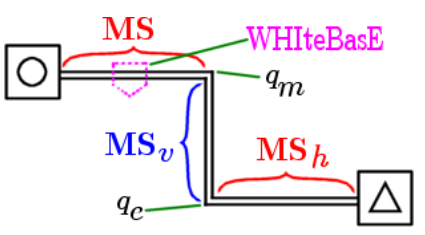

Fig. 37. Names for Double Bend

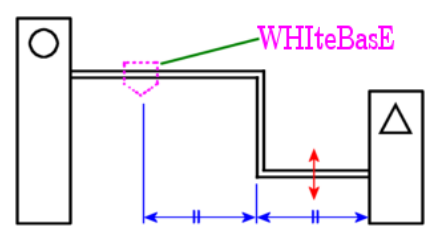

Fig. 38. ON/OFF for Double Bend

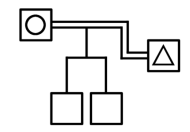

(a)

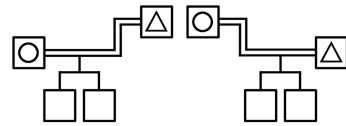

(b) (c)

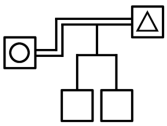

(d)

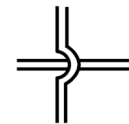

(a)

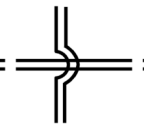

(b)

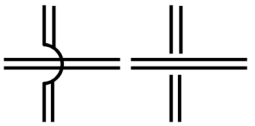

(c)

Fig. 39. Children from Double Bend

\section{Genealogy with Multiple Remarriages}

'Double Bend (DB)' is necessary for displaying multiple remarriages more than $3 \mathrm{x} 3$ partners in 2D. Therefore, MS is extended as shown in Fig. 37, In this case, define that MS denotes the section that WHIteBasE exists, $\mathrm{MS}_{v}$ denotes the vertical section, and $\mathrm{MS}_{h}$ denotes the horizontal section that no WHIteBasE exists. For calculating DB, positions $q_{m}, q_{e}$ are added to Eq. (2). Finally, $\mathbf{Q}$ is represented by

$$
\mathbf{Q}=\left\{q_{b}, q_{L}, q_{R}, q_{d}, q_{a_{j}}, q_{c_{j}}, q_{v l}, q_{v h}, q_{m}, q_{e}, q_{t l}, q_{r b}\right\}
$$

For seamless mouse operations, set the movable range between WHIteBasE and Individual as shown in Fig. 38. When mouse drag vertically in the movable range, MS becomes DB. $\mathrm{MS}_{h}$ can be arbitrarily set both upper side and lower side. In the first dragging operation, the bending positions occurs at the center between WHIteBasE an Individual. After that, $\mathrm{MS}_{v}$ can be arbitrarily moved by using mouse drag operation on the section from WHIteBasE to Individual. If $\mathrm{MS}_{h}$ approaches $\mathrm{MS}, \mathrm{DB}$ return to MS automatically.

WHIteBasE only exists on the section MS when DB is used. Therefore, the connection styles of children from DB have only four patterns as shown in Figs.39. Provided that DB and BSS do not overlap.

The intersection style of DB, that is, crossing between double horizontal segments and double vertical segments, has not been defined in custom. Therefore, some crossing styles can be thought such as shown in Figs.40(a)-(d). For simple viewing, Fig.40(d) is used in this research.

\section{Search Method of Segment intersections}

Using not only regular layout but also AS and DB, there are four horizontal segments and four vertical segments. The positions of segment intersections can be calculated by using only 16 patterns of line crossing as the following: 


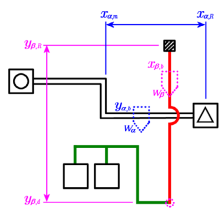

(a) Pattern $1 \mathrm{Aa}$

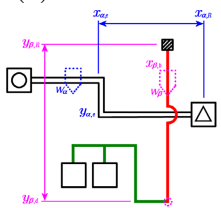

(e) Pattern $1 \mathrm{Ba}$

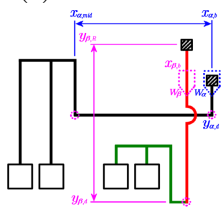

(i) Pattern $3 \mathrm{Aa}$

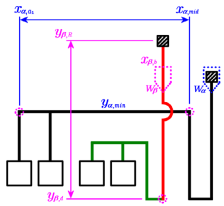

(m) Pattern 3Ba

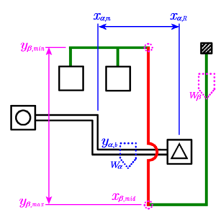

(b) Pattern $1 \mathrm{Ab}$

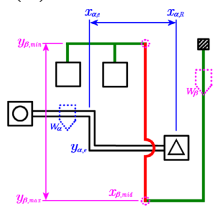

(f) Pattern $1 \mathrm{Bb}$

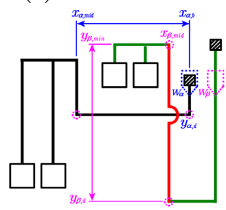

(j) Pattern $3 \mathrm{Ab}$

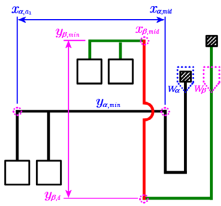

(n) Pattern $3 \mathrm{Bb}$

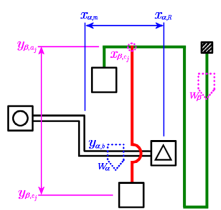

(c) Pattern 2a

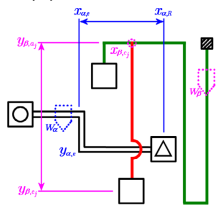

(g) Pattern 2b

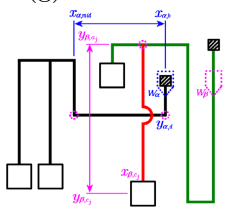

(k) Pattern 4a

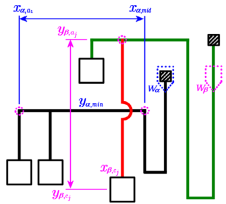

(o) Pattern 4b

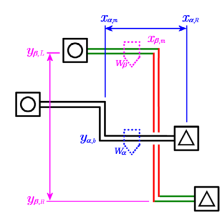

(d) Pattern 5a

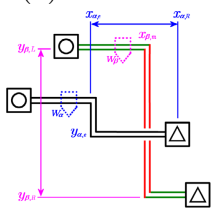

(h) Pattern 5b

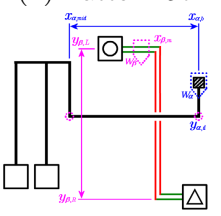

(1) Pattern 6a

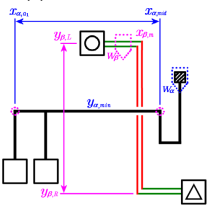

(p) Pattern 6b

Fig. 41. Search Pattern of segment intersections including Double Bend

(a) MS of $W_{\alpha}$ and TS of $W_{\beta}$ are crossing (Fig.41(a))

If $x_{\alpha, m}<x_{\beta, b}<x_{\alpha, R} \& y_{\beta, R}<y_{\alpha, b}<y_{\beta, d}$, the intersection is $\left(x_{\beta, b}, y_{\alpha, b}\right)$.

(b) MS of $W_{\alpha}$ and $\mathrm{BSS}_{v}$ of $W_{\beta}$ are crossing (Fig.41(b))

If $x_{\alpha, m}<x_{\beta, \text { mid }}<x_{\alpha, R} \& y_{\beta, \text { min }}<y_{\alpha, b}<y_{\beta, \max }$, the intersection is $\left(x_{\beta, \text { mid }}, y_{\alpha, b}\right)$ ].

(c) MS of $W_{\alpha}$ and BS of $W_{\beta}$ are crossing (Fig.41(c))

If $x_{\alpha, m}<x_{\beta, c_{j}}<x_{\alpha, R} \& y_{\beta, a_{j}}<y_{\alpha, b}<y_{\beta, c_{j}}$, the intersection is $\left(x_{\beta, c_{j}}, y_{\alpha, b}\right)$.

(d) MS of $W_{\alpha}$ and $\mathrm{MS}_{v}$ of $W_{\beta}$ are crossing (Fig.41(d))

If $x_{\alpha, m}<x_{\beta, m}<x_{\alpha, R} \& y_{\beta, L}<y_{\alpha, b}<y_{\beta, R}$, the intersection is $\left(x_{\beta, m}, y_{\alpha, b}\right)$.

(e) $\mathrm{MS}_{e}$ of $W_{\alpha}$ and TS of $W_{\beta}$ are crossing (Fig.411(e))

If $x_{\alpha, e}<x_{\beta, b}<x_{\alpha, R} \& y_{\beta, R}<y_{\alpha, e}<y_{\beta, d}$, the intersection is $\left(x_{\beta, b}, y_{\alpha, e}\right)$.

(f) $\mathrm{MS}_{e}$ of $W_{\alpha}$ and $\mathrm{BSS}_{v}$ of $W_{\beta}$ are crossing (Fig.41(f))

If $x_{\alpha, e}<x_{\beta, \text { mid }}<x_{\alpha, R} \& y_{\beta, \text { min }}<y_{\alpha, e}<y_{\beta, \text { max }}$, the intersection is $\left(x_{\beta, \text { mid }}, y_{\alpha, e}\right)$.

(g) $\mathrm{MS}_{e}$ of $W_{\alpha}$ and $\mathrm{BS}$ of $W_{\beta}$ are crossing (Fig.411(g))

If $x_{\alpha, e}<x_{\beta, c_{j}}<x_{\alpha, R} \& y_{\beta, a_{j}}<y_{\alpha, e}<y_{\beta, c_{j}}$, the intersection is $\left(x_{\beta, c_{j}}, y_{\alpha, e}\right)$.

(h) $\mathrm{MS}_{e}$ of $W_{\alpha}$ and $\mathrm{MS}_{v}$ of $W_{\beta}$ are crossing (Fig.41(h))

If $x_{\alpha, e}<x_{\beta, m}<x_{\alpha, R} \& y_{\beta, L}<y_{\alpha, e}<y_{\beta, R}$, the intersection is $\left(x_{\beta, m}, y_{\alpha, e}\right)$.

(i) $\mathrm{BSS}_{h}$ of $W_{\alpha}$ and TS of $W_{\beta}$ are crossing (Fig.41(i))

If $x_{\alpha, m i d}<x_{\beta, b}<x_{\alpha, b} \& y_{\beta, R}<y_{\alpha, d}<y_{\beta, d}$, the intersection is $\left(x_{\beta, b}, y_{\alpha, d}\right)$.

(j) $\mathrm{BSS}_{h}$ of $W_{\alpha}$ and $\mathrm{BSS}_{v}$ of $W_{\beta}$ are crossing (Fig.41(j))

If $x_{\alpha, \text { mid }}<x_{\beta, \text { mid }}<x_{\alpha, b} \& y_{\beta, \text { min }}<y_{\alpha, d}<y_{\beta, d}$, the intersection is $\left(x_{\beta, \text { mid }}, y_{\alpha, d}\right)$.

(k) $\mathrm{BSS}_{h}$ of $W_{\alpha}$ and BS of $W_{\beta}$ are crossing (Fig.41(k))

If $x_{\alpha, m i d}<x_{\beta, c_{j}}<x_{\alpha, b} \& y_{\beta, a_{j}}<y_{\alpha, d}<y_{\beta, c_{j}}$, the intersection is $\left(x_{\beta, c_{j}}, y_{\alpha, d}\right)$.

${ }^{1}$ mid denotes $q_{v l}$ or $q_{v h}$, min denotes $q_{v l}$, and max denotes $q_{v h}$. 


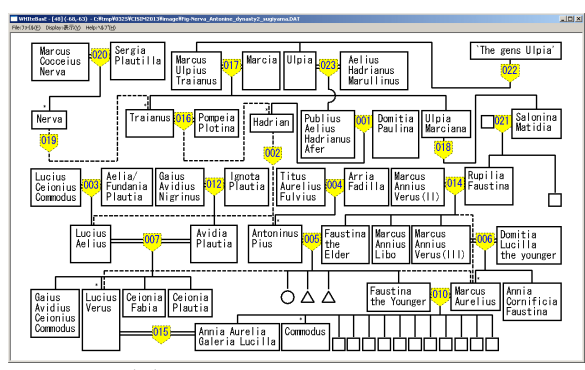

(a) Sample of Adoptions

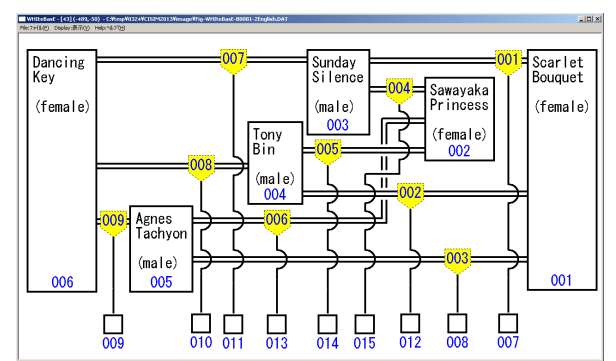

(b) Sample of Multiple Remarriages

Fig. 42. Our New Genealogy Display Software

(l) $\mathrm{BSS}_{h}$ of $W_{\alpha}$ and $\mathrm{MS}_{v}$ of $W_{\beta}$ are crossing (Fig.41(1))

If $x_{\alpha, m i d}<x_{\beta, m}<x_{\alpha, b} \& y_{\beta, L}<y_{\alpha, d}<y_{\beta, R}$, the intersection is $\left(x_{\beta, m}, y_{\alpha, d}\right)$.

(m) $\mathrm{BSS}_{l}$ of $W_{\alpha}$ and TS of $W_{\beta}$ are crossing (Fig.41(m))

If $x_{\alpha, a_{1}}<x_{\beta, b}<x_{\alpha, \text { mid }} \& y_{\beta, R}<y_{\alpha, \min }<y_{\beta, d}$, the intersection is $\left(x_{\beta, b}, y_{\alpha, \text { min }}\right)$.

(n) $\mathrm{BSS}_{l}$ of $W_{\alpha}$ and $\mathrm{BSS}_{v}$ of $W_{\beta}$ are crossing (Fig.41(n))

If $x_{\alpha, a_{1}}<x_{\beta, \text { mid }}<x_{\alpha, \text { mid }} \& y_{\beta, \min }<y_{\alpha, \min }<y_{\beta, d}$, the intersection is $\left(x_{\beta, \text { mid }}, y_{\alpha, \text { min }}\right)$.

(o) $\mathrm{BSS}_{l}$ of $W_{\alpha}$ and $\mathrm{BS}$ of $W_{\beta}$ are crossing (Fig.41(o))

If $x_{\alpha, a_{1}}<x_{\beta, c_{j}}<x_{\alpha, m i d} \& y_{\beta, a_{j}}<y_{\alpha, \min }<y_{\beta, c_{j}}$, the intersection is $\left(x_{\beta, c_{j}}, y_{\alpha, \min }\right)$.

(p) $\mathrm{BSS}_{l}$ of $W_{\alpha}$ and $\mathrm{MS}_{v}$ of $W_{\beta}$ are crossing (Fig.41(p))

If $x_{\alpha, a_{1}}<x_{\beta, m}<x_{\alpha, m i d} \& y_{\beta, L}<y_{\alpha, \min }<y_{\beta, R}$, the intersection is $\left(x_{\beta, m}, y_{\alpha, m i n}\right)$.

If all children of $W_{\beta}$ are adoptions, (a),(b),(e),(f),(i),(j),(m) and (n) are unnecessary to search. If all children of $W_{\alpha}$ are adoptions, (i)-(p) are unnecessary. If the target child of $W_{\beta}$ is adoptions, (c),(g),(k) and (o) are unnecessary.

\section{Demonstration of Our New Software}

Figs.42(a),(b) show the sample demonstrations of our new genealogy display software that can be display Adoptions in (a) and Multiple Remarriages in (b) respectively with segment intersections. Only changing from solid segments to dashed segments can realize adoptions display by using not only regular layout but also DS and HS. Using DB can realize multiple remarriages display. A lot of individuals with complex relations including adoptions could be displayed in narrow area using one window. Even if the client area is filled, scrolling mouse wheel, the zoom rate can be changed and displaying area beyond the window size can be used. Using our method, genealogy required in a favorite rectangle size can be displayed easily. Even if AS and DB are used, segment intersections could be displayed automatically and seamlessly by only mouse operation.

\section{Conclusion}

In this research, Genealogy with Adoptions and Multiple Remarriages could be constructed by using the WHIteBasE method. Future research will be conducted to construct automated layouts, generation search, thinned-out individuals, grid layouts, improving GUI, etc. This research has received the assistance of the "Shin Buddhist Comprehensive Research Institute, Otani University, Japan". 


\section{References}

1. Sugito, S.: "Alliance", news letter of Oceania conference, vol. (86), pp. 10-37 (2006) (in Japanese)

2. The Generations Network. Genealogy, Family Trees and Family History Records on line, http://ancestry.com

3. MyHeritage, http://myheritage.jp

4. Jurek Software, http://www.pedigree-draw.com/

5. He, M., Li, W.: PediDraw: A web-based tool for drawing a pedigree in genetic counseling. In: BMC Medical Genetics, pp. 1-4 (2007)

6. Brun-samarcq, L., et al.: CoPE: a collaborative pedigree drawing environment. Bioinformatics 'Applications Note' 15(4), 345-346 (1999)

7. Dudbridge, F., et al.: Pelican: pedigree editor for linkage computer analysis. Bioinformatics 'Applications Note' 20(14), 2327-2328 (2004)

8. Trager, E.H., et al.: Madeline 2.0 PDE: a new program for local and web-based pedigree drawing. Bioinformatics 'Applications Note' 23(14), 1854-1856 (2007)

9. Makinen, V.P., et al.: High-throughput pedigree drawing. European Journal of Human Genetics 13, 987-989 (2005)

10. Mancosu, G., Ledda, G., Melis, P.M.: PedNavigator: a pedigree drawing servlet for large and inbred populations. Bioinformatics 'Applications Note' 19(5), 669-670 (2003)

11. Tores, F., Barillot, E.: The art of pedigree drawing: algorithmic aspects. Bioinformatics 17(2), 174-179 (2001)

12. Loh, A.M., et al.: Celestial3D: a novel method for 3D visualization of familial data. Bioinformatics 'Applications Note' 24(9), 1210-1211 (2008)

13. Aida, M.: Construction of a Japanese classic genealogy database. IPSJ SIG Computers and the Humanities, 2001-CH-051-6, 39-46 (2001) (in Japanese)

14. Bennett, R.L., et al.: Recommendations for Standardized Human Pedigree Nomenclature. Journal of Genetic Counseling 4(4), 267-279 (1995)

15. PED Pedigree Software, http://www.medgen.de/ped/

16. PAF, http://www.familysearch.org/

17. ScionPC, http://homepages.paradise.net.nz/scionpc/

18. XY Family Tree, http://www.xy-family-tree.com/

19. WeRelate, http://www .werelate.org/wiki/Main_Page/

20. GenoPro, http://www.genopro.com/

21. Naito, M.: Topic Map for Displaying Genealogy. SIG-SWO-A603-04, pp. 4-1-4-7 (2007) (in Japanese)

22. GEDCOM LETTER, http://en.wikipedia.org/wiki/GEDCOM

23. Sugiyama, S., Ikuta, A., Shibata, M., Matsuura, T.: A Study of an Event Oriented Data Management Method for Displaying Genealogy: Widespread Hands to InTErconnect BASic Elements (WHIteBasE). International Journal of Computer Information Systems and Industrial Management Applications (IJCISIM), 280-289 (2011) ISSN: 2150-7988/2

24. Sugiyama, S., Ikuta, A., Yokozawa, D., Shibata, M., Matsuura, T.: Displaying Genealogy with Various Layouts by using the "WHIteBasE" Method. International Journal of Computer Information Systems and Industrial Management Applications (IJCISIM), 102-115 (2014), ISSN: 2150-7988/6

25. Nerva-Antonine dynasty, http://en.wikipedia.org/wiki/Nerva

26. Racehorses, http://db.netkeiba.com/?pid=horse_top (in Japanese) 